\title{
LncRNA BCYRN1 promotes cell migration and invasion of non-small cell lung cancer via the miR-30b-3p/ROCK1 axis
}

\author{
Shu SONG ${ }^{1, *}$, Wei-Gang BIAN ${ }^{2, *}$, Zhu QIN, Dong ZENG ${ }^{1}$, Jing-Jing XU1, Hai-Cheng TANG ${ }^{3, *}$ \\ ${ }^{1}$ Department of Pathology, Shanghai Public Health Clinical Center, Fudan University, Shanghai, China; ${ }^{2}$ Department of Oncology, The First \\ People's Hospital of Yancheng City, Yancheng, China; ${ }^{3}$ Department of Respiratory Medicine, Shanghai Public Health Clinical Center, Fudan \\ University, Shanghai, China
}

*Correspondence: tanghaicheng1012@163.com

"Contributed equally to this work.

Received July 26, 2021 / Accepted January 21, 2022

\begin{abstract}
Long non-coding RNA brain cytoplasmic RNA 1 (LncRNA BCYRN1) has been proved to participate in the cancer cell metastasis process, including non-small cell lung cancer (NSCLC). However, the underlying molecular mechanisms involved in the BCYRN1-mediated function remain largely unknown. The qRT-PCR analysis was carried out to examine the relative expressions of BCYRN1, microRNA-30b-3p (miR-30b-3p), and Rho-associated coiled-coil protein kinase 1 (ROCK1). ROCK1 protein level was detected via western blot assay. The migrative and invasive abilities of H520 and A549 cells were evaluated via Transwell assay. The relationships between BCYRN1 and miR-30b-3p or ROCK1 and miR-30b-3p were examined by luciferase reporter assay. The expression levels of BCYRN1 and ROCK1 were upregulated in NSCLC tissues and cells, while miR-30b-3p was downregulated. Higher BCYRN1 expression indicated lymph node metastasis and advanced tumor-node-metastasis (TNM) stage of NSCLC patients. Loss of BCYRN1 suppressed cell migration and invasion. More importantly, miR-30b-3p possessed the binding sites with BCYRN1. Besides, BCYRN1 negatively regulated the expression level of miR-30b-3p. Meanwhile, ROCK1 was proven to be directly targeted by miR-30b-3p. In addition, the silencing of miR-30b-3p also weakened the effect of BCYRN1 knockdown on cell migration and invasion. In vivo, BCYRN1 silencing reduced the growth of A549 cells. LncRNA BCYRN1 was involved in the metastasis of NSCLC through modulating the miR-30b-3p/ROCK1 axis.
\end{abstract}

Key words: LncRNA BCYRN1; NSCLC; metastasis; miR-30b-3p; ROCK1

Non-small cell lung cancer (NSCLC) is the leading cause of cancer-related death worldwide [1,2]. Although many researchers have made a remarkable effort in treating NSCLC patients, the overall 5-year survival rate of this disease remained low. Therefore, a deep understanding of molecular mechanisms, which contribute to NSCLC progression could provide more effective diagnostic markers for NSCLC patients.

Long non-coding RNAs (lncRNAs) are discovered to function in cancer carcinogenesis and progression [3-6], as well as human disease, including abdominal aortic aneurysms [7], glioblastoma [8], breast cancer, and NSCLC [9-11]. LncRNA GIAT4RA has been shown to play a critical role as a ubiquitination regulator and tumor suppressor in NSCLC [12]. LncRNA 00312 was reported to inhibit NSCLC cell proliferation and promote apoptosis [13]. He et al. [14] revealed that FEZF1-AS1 is upregulated in NSCLC tissues, implying lymph node metastasis, poor differentiation grade, and advanced tumor-node-metastasis (TNM) stage. They further disclosed that depletion of FEZF1-AS1 suppresses cell proliferation, invasion, and epithelial-mesenchymal transition (EMT) process in NSCLC. In addition, the lncRNA-microRNA-mRNA interaction is widely linked with the modulation of cancers [15]. In another study, it reported that MALAT1 is evidently upregulated in human NSCLC cell lines including A549, H23, H522, H1299, and H460. Moreover, knockdown of MALAT1 induces the inhibition of cell proliferation, colony formation, and apoptosis via regulating the miR-124/STAT3 axis [16]. The previous study also indicated that the CCAT1/miR-130a-3p/SOX4 axis is required for the cisplatin resistance of NSCLC cells [17]. Recently, BCYRN1 has been found to promote NSCLC cell proliferation and metastasis [18]. Although studies have explored the roles of BCYRN1, the molecular mecha- 
nisms of BCYRN1 have not been elaborated. Researchers have revealed that downregulated miR-30b expression can inhibit cell proliferation in NSCLC [19]. Furthermore, it is well documented that ROCK1 is widely highly expressed in NSCLC, which is associated with lymph node metastasis [20]. However, the direct evidence of the involvement of miR-30b-3p and ROCK1 in the regulation of BCYRN1 in NSCLC metastasis is still lacking.

In this study, we sought to investigate the function of BCYRN1 in regulating cell migration and invasion and address the role of the BCYRN1/miR-30b-3p/ROCK1 axis in NSCLC.

\section{Patients and methods}

Clinical samples. 42 paired NSCLC tissues have been obtained from the Shanghai Public Health Clinic Center. Meanwhile, the informed consent was signed by all patients. The experiments were approved by the Ethics Committee of Shanghai Public Health Clinic Center. Besides, the clinicopathological features of NSCLC patients are displayed in Table1.

Cell culture and transfection. NSCLC cell lines A549, NCI-H1650, NCI-H1299, H520 and human lung epithelial cell lines HBE, 16HBE, BEAS-2B were obtained from the American Type Culture Collection (Manassas, VA, USA). Cells were maintained in Dulbecco's modified Eagle's medium (Sigma, St. Louis, MO, USA) with additional 10\% fetal bovine serum (FBS; Invitrogen; Carlsbad, CA, USA) and $1 \%$ penicillin/streptomycin (Sigma) solution, in a humidified atmosphere containing $5 \% \mathrm{CO}_{2}$ at $37^{\circ} \mathrm{C}$. Small interfering RNA for BCYRN1 (si-BCYRN1) or its negative

Table 1. Correlation between lncRNA BCYRN1 expression and clinicopathological features in NSCLC patients.

\begin{tabular}{|c|c|c|c|}
\hline \multirow{2}{*}{ Parameters } & \multicolumn{2}{|c|}{ BCYRN1 expression } & \multirow{2}{*}{ p-value } \\
\hline & High $(n=22)$ & Low $(n=20)$ & \\
\hline Age & & & 0.976 \\
\hline$<60$ years & 10 & 9 & \\
\hline$\geq 60$ years & 12 & 11 & \\
\hline Gender & & & 0.217 \\
\hline Female & 9 & 12 & \\
\hline Male & 13 & 8 & \\
\hline Differentiation & & & 0.951 \\
\hline Well & 4 & 3 & \\
\hline Moderate & 10 & 9 & \\
\hline Poor & 8 & 8 & \\
\hline Lymph node metastasis & & & 0.008 \\
\hline Yes & 20 & 11 & \\
\hline No & 2 & 9 & \\
\hline TNM stage & & & 0.001 \\
\hline I-II & 4 & 14 & \\
\hline III & 18 & 6 & \\
\hline
\end{tabular}

control (si-NC), miR-30b-3p inhibitor (anti-miR-30b-3p) or its negative inhibitor (anti-miR-NC) were introduced into H520 or A549 cells.

Transient RNA interference. Small interfering RNA (si-RNA) sequences for targeting ROCK1 and BCYRN1 were as follows: ROCK1 (Homo-3354) 5'-GCAGAUGAAACAGGAAAUATTUAUUUCCUGUUUCAUCUGCTT-3'; si-BC YRN1 5'-CUCCAGAAAAAGGAAAAAAAAAA-3' (Gene Pharma, Shanghai, China). H520 and A549 cells were plated in a six-well plate. On day 2 , when cells reach $40-60 \%$ density, we transiently cotransfected with 100 pmol of si-ROCK1 or si-NC sequences using $5 \mu$ Jet PRIME (Polyplus, IIIkirch, France). Four hours later, we replaced the supernatant with a fresh medium to culture the cells for an additional 48-72 h.

RNA extraction, cDNA synthesis, and qRT-PCR assay. Total RNA from cells or tissues was extracted using Trizol reagent (Sigma). TaqMan ${ }^{\star}$ MicroRNA Reverse Transcription kit (Biosystems, Forster City, CA, USA) was used to synthesize complementary DNA (cDNA). All primers were listed as follows: BCYRN1-F 5'-CTGGGCAATATAGCGAGAC-3', BCYRN1-R 5' -TGCTTTGAGGGAAGTTACG-3'; ROCK1-F 5'-AACCATGTGACTGAGTGCCC-3', ROCK1-R 5'-TCAGTGTGTTGTGCCAAAGC-3'; GAPDH-F 5' - AGAAGGCTGGGGCTCATTTG-3'; GAPDH-R 5'-AGGGGCCATCCACAGTCTTC-3'; miR-30b-3p-F 5'-GCGGCGGTGTAAACATCCTACAC-3', miR-30b3p-R 5'-ATCCAGTGCAGGGTCCGAGG-3'; U6-F 5'-CTCGCTTCGGCAGCACA-3', U6-R 5'-AACGCTTCACGAATTTGCGT-3'. The relative miR-30b-3p expression and BCYRN1 or ROCK1 expression (normalized to U6 and GAPDH separately) were analyzed by the $2^{-\Delta \Delta \mathrm{Ct}}$ method.

Transwell assay. Cell migration and invasion assays were carried out in a 24-well Transwell plate (Corning, NY, USA). The upper wells of the Transwell inserts were filled with a cell suspension $\left(1 \times 10^{5} / \mathrm{ml}\right)$ and $15 \%$ FBS $(500 \mu \mathrm{l})$ was added into the lower chamber. For invasion assay, Matrigel (Sigma) coated the upper chamber for $2 \mathrm{~h}$ before we perform the Transwell assay. After overnight incubation, all migratory and invasive cells on the bottom of chambers were fixed with methanol and stained with crystal violet (Sigma). The numbers of migrated cells were counted in 3 random views under a microscope (40× magnification; BX51; Olympus Corporation, Tokyo, Japan).

Western blot assay. After transfection, cells were harvested and lysed, separated by sodium dodecyl sulfatepolyacrylamide gel electrophoresis, and then transferred to polyvinylidene difluoride membranes (Millipore, Billerica, MA, USA). The membranes were blocked with 5\% skim milk overnight, then blotted with primary antibodies (antiROCK1, ab97592, Abcam; anti-F-actin, ab205, Abcam; antiGAPDH, ab128915, Abcam) at $4{ }^{\circ} \mathrm{C}$ for overnight and then incubated with horseradish peroxidase-conjugated (HRP) secondary antibody (ab205718, Abcam) for $1 \mathrm{~h}$. The chemiluminescence kit (Beyotime, Shanghai, China) was selected for signal detection. 
Luciferase reporter assay. The 3'-UTR of BCYRN1 was cloned into the downstream region of the firefly luciferase located in the pMIR-GLO ${ }^{\mathrm{m}}$ Luciferase vector (Thermo Fisher Scientific) to construct the wild-type (wt) or mutant (mut) of BCYRN1. Similarly, the wild-type 3'-UTR region of ROCK1 carrying a putative miR-30b-3p binding site was introduced into the psi-CHECK2 vector. And we also synthesized the ROCK1-mutant plasmid. H520 and A549 cells cultured in 24-well plates were co-transfected with BCYRN1-wt, BCYRN1-mut, ROCK1-wt, or ROCK1-mut plasmids along with miR-30b-3p mimic or NC. Luciferase activities were analyzed $48 \mathrm{~h}$ after transfection using a Dual-Luciferase Reporter Assay System (Promega, Madison, WI, USA).

Xenograft tumor model. For the in vivo tumorigenesis experiments, $\mathrm{BALB} / \mathrm{c}$ nude mice (females, 6-8 weeks old, $\mathrm{n}=3$ /group) were purchased from the Shanghai Animal Experiment Center (Shanghai, China) and randomly divided into two groups. All animal experiments were approved by the animal ethics committee of the Shanghai Public Health Clinic Center. The shRNA lentiviral vectors for BCYRN1 (sh-BCYRN1) and its negative control (sh-NC) were obtained from GenePharma (Shanghai, China). $2 \times 10^{6}$ A549 cells stably transfected with sh-BCYRN1 or sh-NC were injected subcutaneously into the right side of nude mice to construct a nude-mouse transplantation model. Tumor size was recorded every seven days and volume was calculated according to the formula $=0.5 \times$ length $\times$ width $^{2}$. After 28 days, nude mice were euthanized and tumors were weighed immediately after removal. The expression of BCYRN1 and miR-30b-3p was analyzed by qRT-PCR.

Immunohistochemistry (IHC). Surgically resected tumor tissue was sectioned $(6 \mu \mathrm{m})$ and immunostained. Sections were dewaxed in xylene, then hydrated in gradient alcohol, and finally washed three times with PBS. Tissue sections were incubated with anti-ROCK1 (1:100, ab97592, Abcam, Cambridge, UK) for $60 \mathrm{~min}$ at room temperature. Biotinylated goat anti-rabbit serum IgG (A7016, Beyotime
Institute of Biotechnology) was used as a secondary antibody. Antigen/antibody complexes were visualized with diaminobenzidine (DAB; Dako, Carpinteria, CA, USA) and re-stained with hematoxylin. The images were analyzed using the IMS imaging processing system.

Statistical analysis. The experiment was repeated three times. All data in this study were shown as mean \pm standard deviation (SD). The Student's t-test or one-way analysis of variance (ANOVA) analyses were evaluated by SPSS 20.0 version (SPSS, Chicago, IL, USA). A p-value $<0.05$ was considered to be statistically significant.

\section{Results}

BCYRN1 is upregulated in NSCLC tissues and cells, and this upregulation is linked to the poor prognosis of NSCLC patients. We obtained 42 paired tissues from NSCLC patients and examined the BCYRN1 expression via qRT-PCR assay first. And data showed upregulated BCYRN1 expression in NSCLC tissues when compared to the adjacent normal lung tissues (Figure 1A). Further analysis of clinicopathological features demonstrated that the high expression level of BCYRN1 in NSCLC tissues contributed to the advanced TNM stage and lymph node metastasis (Table 1). Subsequently, we further examined the expression of BCYRN1 in NSCLC cells. Data showed that the BCYRN1 level was higher in NCI-H1650, NCI-H1299, A549, and H520 cells than that of $16 \mathrm{HBE}, \mathrm{HBE}$, and BEAS-2B cells (Figure 1B). Those data indicated that BCYRN1 may contribute to NSCLC development.

Knockdown of BCYRN1 inhibits the malignant progression of NSCLC cells. Then, a loss-of-function experiment was performed to evaluate the effect of BCYRN1 knockdown on metastasis of NSCLC. The transfection efficiency revealed that the introduction of si-BCYRN1 obviously decreased BCYRN1 expression in both H520 and A549 cells (Figures 2A, 2B). Moreover, si-RNA-mediated downregu-
A

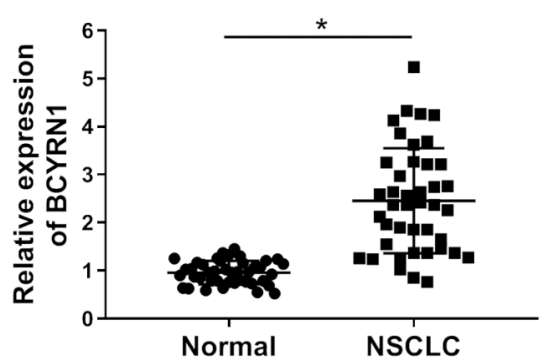

B

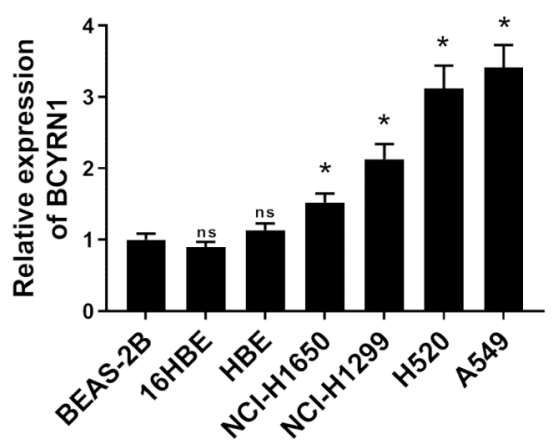

Figure 1. The expression of BCYRN1 in NSCLC tissues and cells. A) The expression of BCYRN1 in NSCLC tissues and matched adjacent normal tissues. B) The level of BCYRN1 in NSCLC adenocarcinoma cell lines NCI-H1650, NCI-H1299, and A549, squamous lung carcinoma cell line H520, and human lung epithelial cell lines HBE, $16 \mathrm{HBE}$, and BEAS-2B. The experiment was repeated three times. ${ }^{*} \mathbf{p}<0.05$ 

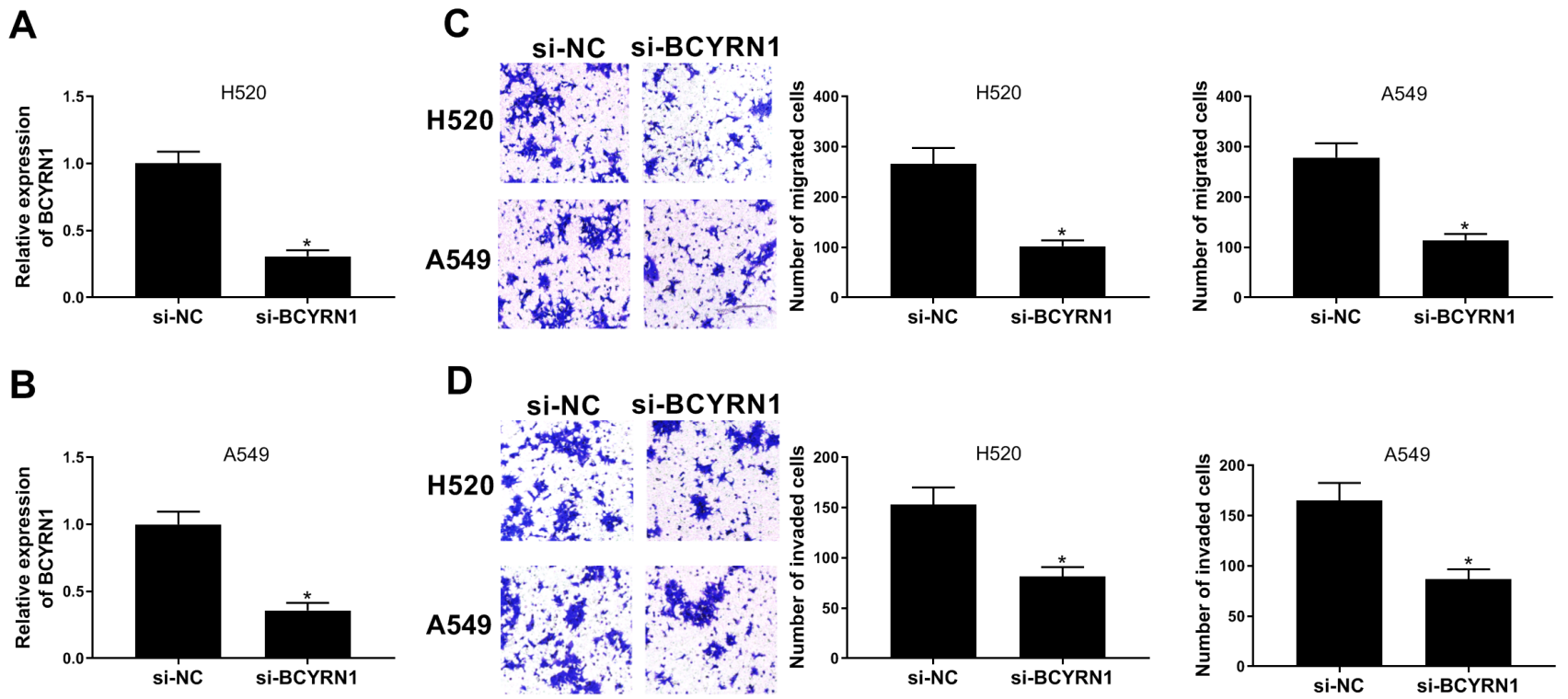

Figure 2. The effect of BCYRN1 knockdown on migration and invasion of NSCLC. A, B) H520 and A549 cells were transfected with si-BCYRN1 and si-NC, respectively. C, D) Transwell assay was performed to evaluate cell migration and invasion. The experiment was repeated three times. ${ }^{*} \mathrm{p}<0.05$

A

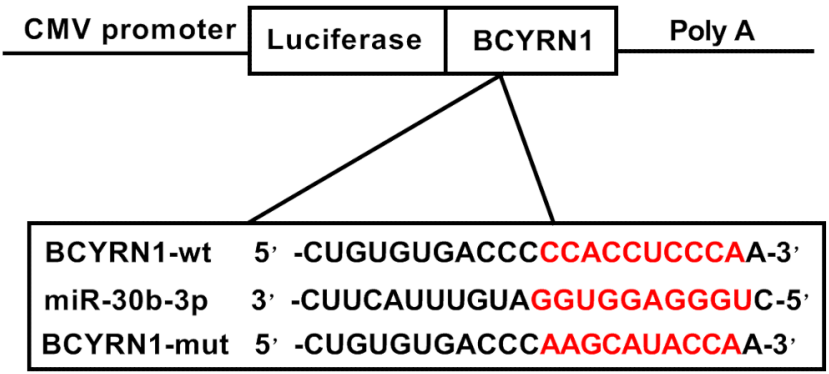

B
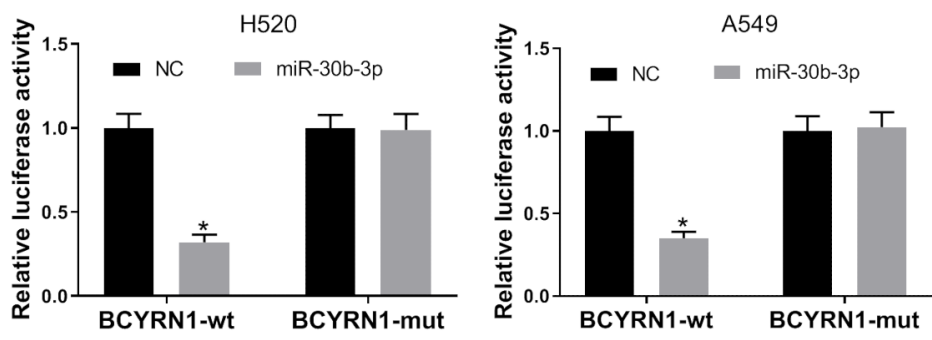

Figure 3. BCYRN1 interacts with miR-30b-3p. A) The binding sites between miR-30b-3p and BCYRN1. B) Luciferase reporter assay was carried out to determine the relationship between miR-30b-3p and BCYRN1. The experiment was repeated three times. ${ }^{\star} \mathrm{p}<0.05$

lation of BCYRN1 markedly inhibited the migrative and invasive ability of NSCLC cells (Figures 2C, 2D). Therefore, we thought that a high level of BCYRN1 in NSCLC may contribute to the metastasis of NSCLC.

BCYRN1 negatively regulates the expression of miR-30b-3p. LncRNAs usually could act as competitive endogenous RNAs (ceRNAs) of miRNAs. In our study, we further found that BCYRN1 contained binding sites with
miR-30b-3p using DIANA tools (Figure 3A). Meanwhile, luciferase activity was also examined in cells co-transfected with BCYRN1-mut or BCYRN1-wt and miR-30b-3p mimic or NC. An evident decrease of luciferase activity was detected in cells co-transfected with BCYRN1-wt plasmid and miR-30b-3p mimic. However, there was no obvious change in the BCYRN1-mutant group (Figure 3B). After that, we found decreased miR-30b-3p expression in NSCLC tissues 
and cells (Figures 4A, 4B). More interestingly, a negative correlation between miR-30b-3p and BCYRN1 was observed in NSCLC tissues (Figure 4C). Importantly, overexpression of BCYRN1 notably repressed the level of miR-30b-3p in H520 and A549 cells (Figure 4D). Whereas, the BCYRN1 ablation enhanced miR-30b-3p expression (Figure 4E). The above results indicated that miR-30b-3p may be involved in the effect of BCYRN1 on metastasis of NSCLC.

miR-30b-3p inhibits the malignant progression of NSCLC through ROCK1. miRNAs could modulate gene expression. The public database showed that miR-30b-3p can potentially target ROCK1 (Figure 5A). Meanwhile, the lucif-
A

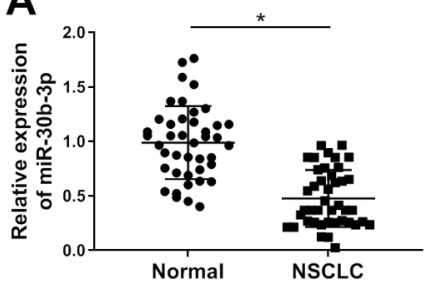

D

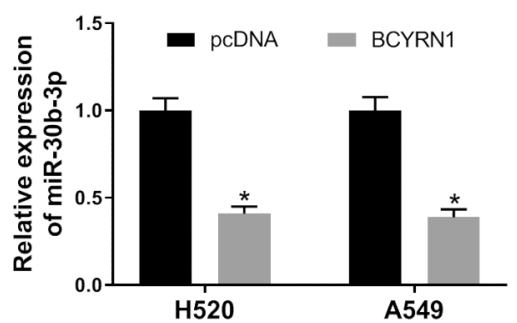

B

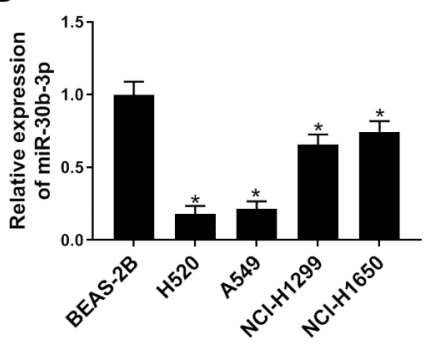

C

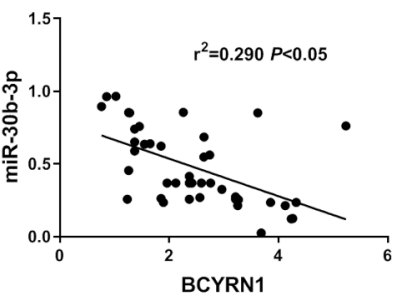

E

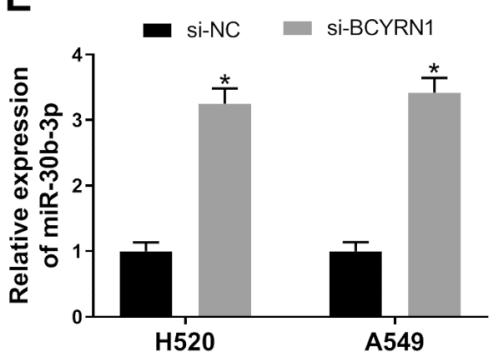

Figure 4. The expression of miR-30b-3p is negatively correlated with BCYRN1. A) The level of miR-30b-3p in NSCLC tissues and matched adjacent normal tissues. B) The level of miR-30b-3p in NCI-H1650, NCI-H1299, A549, H520, and BEAS-2B cells. C) The correlation between miR-30b-3p and BCYRN1 in NSCLC tissues. D) The expression of miR-30b-3p in cells introduced with pcDNA3.0 vector or BCYRN1 overexpression plasmid. E) The expression of miR-30b-3p in cells transfected with si-NC or si-BCYRN1. The experiment was repeated three times. ${ }^{*} \mathbf{p}<0.05$

A

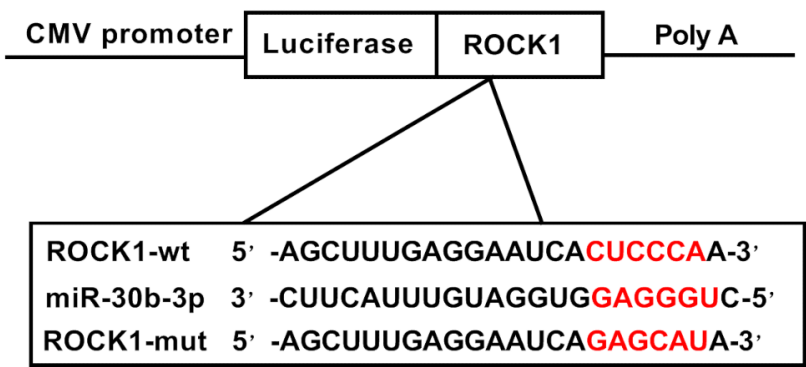

B
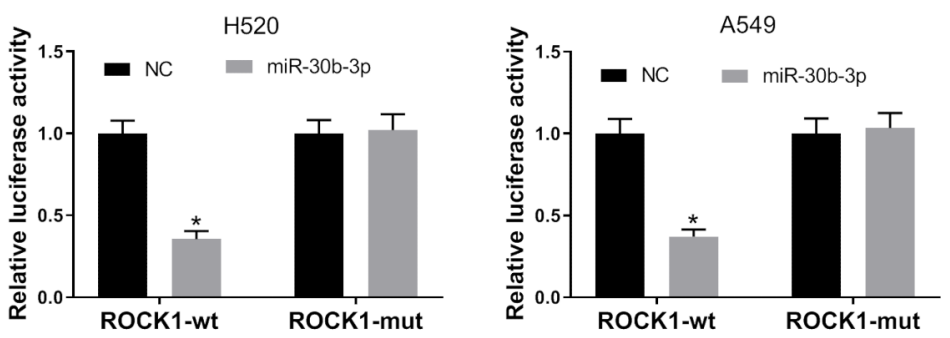

Figure 5. ROCK1 is a target of miR-30b-3p. A) The binding sites between miR-30b-3p and ROCK1. B) The luciferase reporter assay was carried out to determine the relationship between miR-30b-3p and ROCK1. The experiment was repeated three times. ${ }^{*} p<0.05$ 
erase reporter assay also verified the relationship between them (Figure 5B). Similar to that of BCYRN1, the abundance of ROCK1 was also increased in NSCLC tissues and cells (Figures 6A, 6B). Correlation assay showed that ROCK1 expression was negatively correlated with that of miR-30b-3p (Figure 6C). As expected, the protein level of ROCK1 was inhibited in miR-30b-3p overexpression-mediated cells (Figure 6D), while it was promoted in cells introduced with miR-30b-3p inhibitor (Figure 6E), implying that ROCK1 can be directly targeted by miR-30b-3p in NSCLC.

LncRNA BCYRN1 participates in the metastasis of NSCLC through the miR-30b-3p/ROCK1 axis. Finally, we carried out the rescue-of-function experiment to further verify the role of the BCYRN1/miR-30b-3p/ROCK1 axis in NSCLC. The results of western blot revealed that silencing of BCYRN1 curbed the protein expression of ROCK1, which was undermined by the anti-miR-30b-3p transfection (Figures 7A, 7B). Cancer metastasis involves aberrant expression of a range of cytoskeletal proteins, ultimately leading to actin reorganization and migration of cancer cells.
To determine whether F-actin, a downstream effector protein of ROCK1, plays a role in BCYRNA1-mediated NSCLC cell migration and apoptosis, this study also focused on the ROCK1/F-actin axis. Western blotting showed that knockdown of BCYRN1 enhanced the ROCK1 expression, followed by a decrease in F-actin expression. This result suggested that low expression of BCYRN1 maintained F-actin homeostasis, and this effect was reversed by anti-miR-30b-3p (Figures 7C, 7D). These observations indicated that BCYRN1 might regulate NSCLC cell migration through the ROCK1/Factin pathway. Transwell assay further disclosed that the absence of miR-30b-3p also receded the BCYRN1 depletionmediated impairment on cell migration (Figure 7E). Besides, cell invasion was impeded in the si-BCYRN1 group, while the introduction of anti-miR-30b-3p rescued this inhibition effect (Figure 7F). In conclusion, our data implicated that BCYRN1 downregulation hampered cell metastasis via modulating the miR-30b-3p/ROCK1 axis.

Downregulation of IncRNA BCYRN1 inhibits tumor progression in vivo. To further determine the tumori-

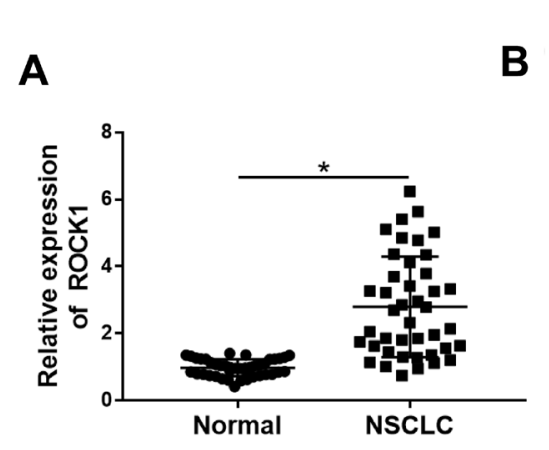

D

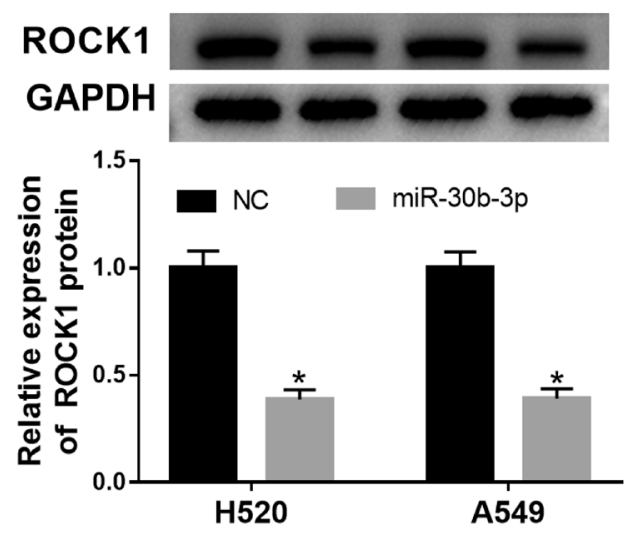

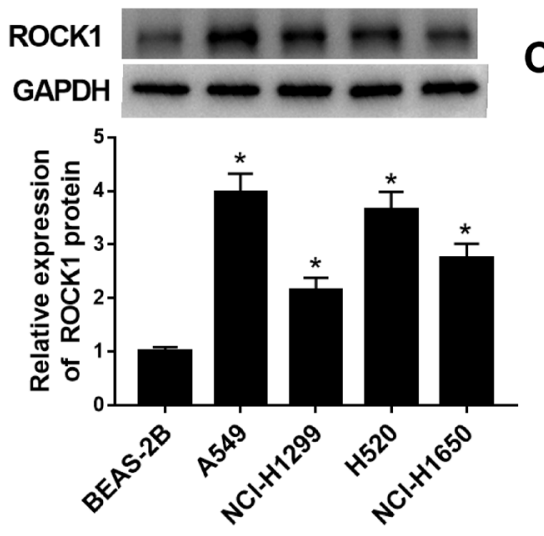

C

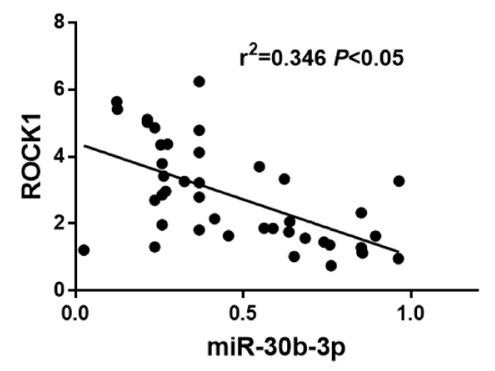

E

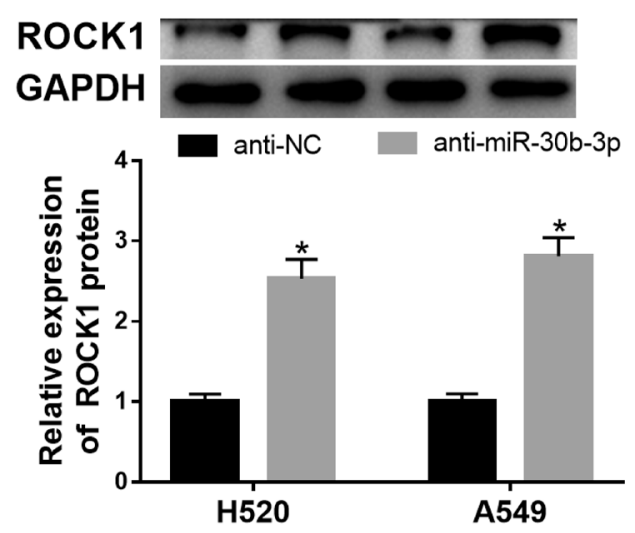

Figure 6. The expression of miR-30b-3p is negatively correlated with ROCK1. A) The level of ROCK1 in NSCLC tissues and matched adjacent normal tissues. B) The level of ROCK1 in NCI-H1650, NCI-H1299, A549, H520, and BEAS-2B cells. C) The correlation between miR-30b-3p and ROCK1 in NSCLC tissues. D) The expression of ROCK1 in cells introduced with miR-30b-3p mimic (miR-30b-3p) or its negative control (NC). E) The expression of ROCK1 in cells transfected with miR-30b-3p inhibitor (anti-miR-30b-3p) or its negative control (anti-NC). The experiment was repeated three times. ${ }^{\star}$ p $<0.05$ 
A
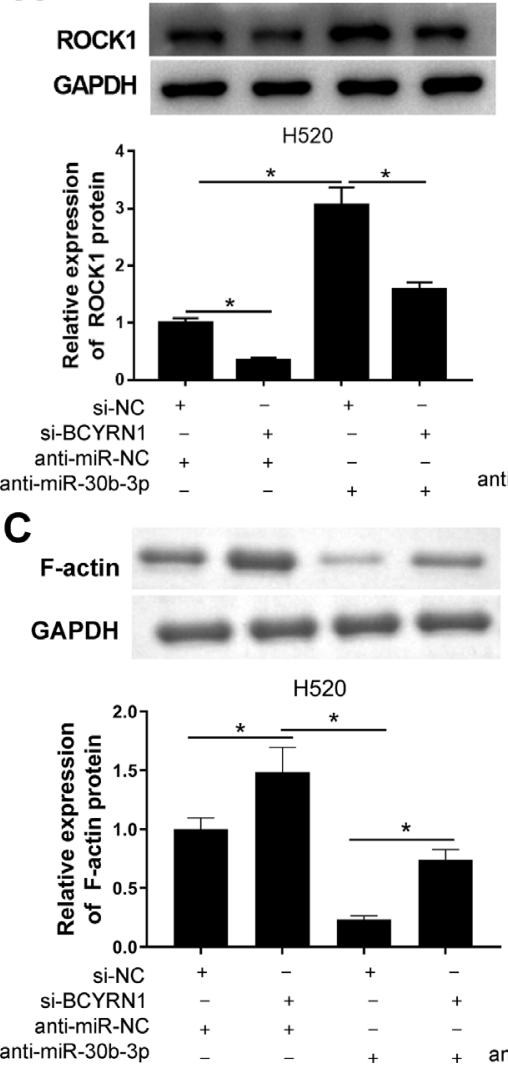

B
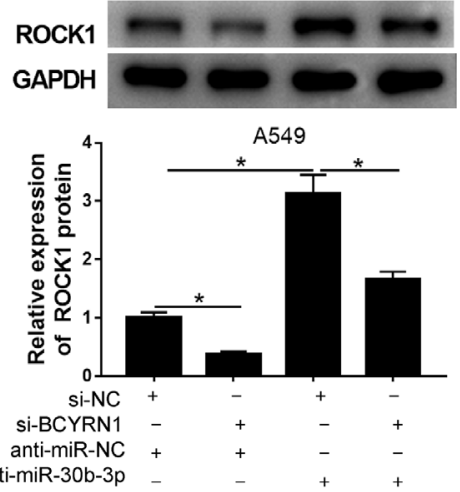

D

F-actin

GAPDH

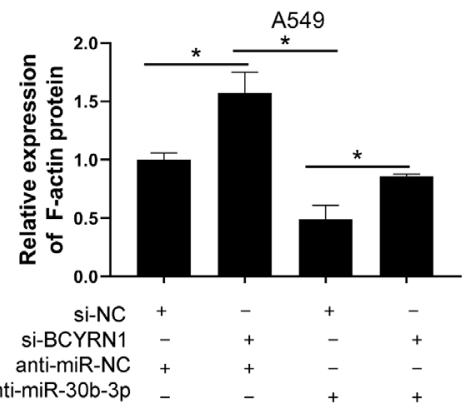

E

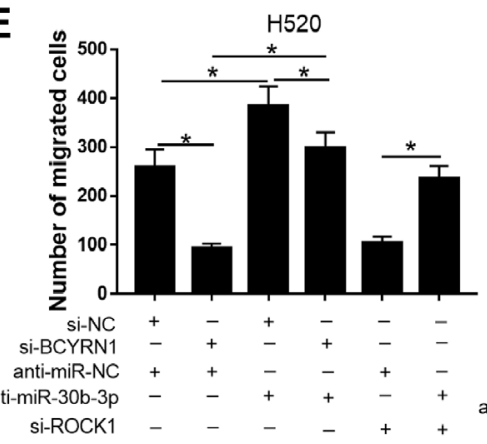

$\mathbf{F}$

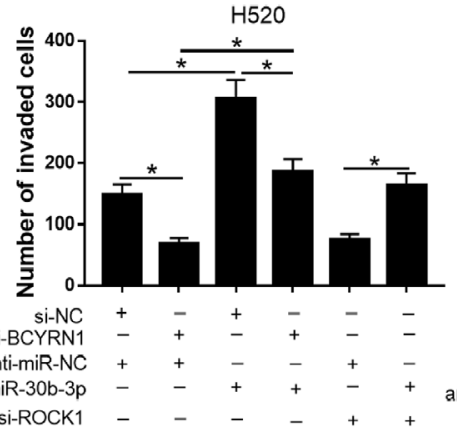

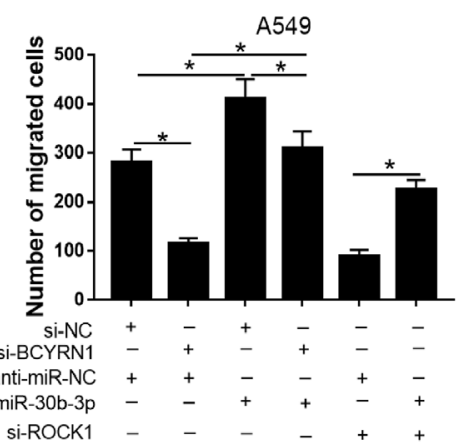

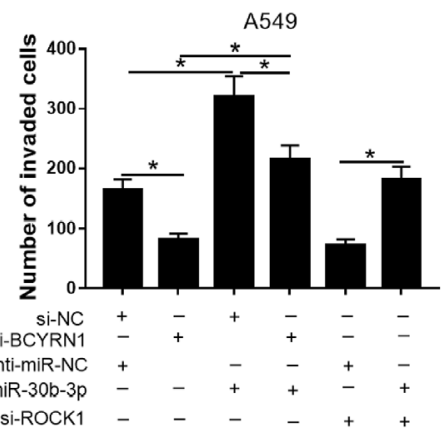

Figure 7. BCYRN1 is required for the metastasis of NSCLC via the miR-30b-3p/ROCK1 axis. A, B) Western blot was used to detect the protein level of ROCK1 in cells co-transfected with si-BCYRN1 and anti-miR-30b-3p. C, D) Western blot was conducted to analyze the expression of F-actin, a downstream target protein of ROCK1, in A549 and H520 cells. E, F) Transwell assay was performed to evaluate cell migration and invasion. The experiment was repeated three times. ${ }^{*} \mathbf{p}<0.05$ 
genic ability of BCYRN1 in vivo, A549 cells with or without BCYRN1 knockdown were injected subcutaneously into nude mice to establish a xenograft tumor model. Tumors in the sh-BCYRN1 group were smaller in size and weight and grew more slowly compared to the sh-NC group (Figures $8 \mathrm{~A}$, $8 \mathrm{~B})$. The expression of BCYRN1 and miR-30b-3p in each tumor was analyzed by qRT-PCR, and the results confirmed that silencing of BCYRN1 largely promoted the expression of miR-30b-3p (Figures 8C, 8D). IHC analysis of tissue specimens showed that ROCK1 expression was significantly repressed in tumor tissues derived from the sh-BCYRN1 group (Figure $8 \mathrm{E}$ ). These results implicated that inhibition of BCYRN1 impeded the tumorigenic ability of NSCLC cells in vivo.

\section{Discussion}

Long non-coding RNAs are associated with a variety of biological roles in cells and regulate disease progression through these effects, such as involvement in cell senescence [21], cell apoptosis [22], and cell proliferation, as well as maintaining cellular genomic stability [23]. Mounting evidence has reported that lncRNAs are implicated in the process of NSCLC [24-26]. BCYRN1 has been widely proved to function as an oncogene in various kinds of cancers. $\mathrm{Gu}$ et al. [27] revealed that BCYRN1 is elevated in colorectal cancer (CRC) tumor tissues. They further demonstrated that the high expression of BCYRN1 is related to tumor size and pathological stages of CRC patients. Moreover, their results also provided evidence that depletion of BCYRN1 induces proliferation inhibition and apoptosis promotion. In cervical cancer, higher BCYRN1 expression also enhances cell proliferation and metastasis via sponging miR-138 [28]. In gastric carcinoma, BCYRN1 is also upregulated in vivo, and silencing of BCYRN1 hampers cell proliferation and migration abilities and blocked the G1/G0 cell cycle, while induced apoptosis in vitro [29]. In this study, we disclosed that lncRNA BCYRN1 was indeed upregulated in NSCLC tissues and cells, which was similar to a previously published study [30]. Moreover, the knockdown of BCYRN1 can suppress the ability of cell migration and invasion. The further analysis of the correlation between BCYRN1 expression and clinicopathological features indicated that the high expression of BCYRN1 was associated with lymph node metastasis and the high TNM stage. Those data suggested that BCYRN1 may be an important potential therapeutic target of NSCLC.

More importantly, lncRNAs are shown to function as ceRNAs of miRNAs to modulate gene expression, thus participating in the biological process in various diseases. In our report, miR-30b-3p was proved to sponge with BCYRN1 using a luciferase reporter assay. Furthermore, miR-30b-3p was significantly declined in both NSCLC tissues and cells. It also presented a negative correlation
A

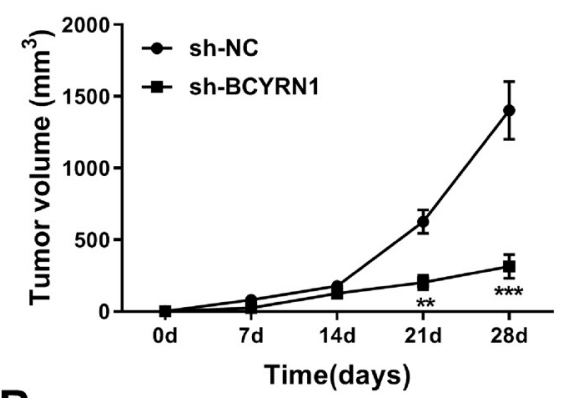

B

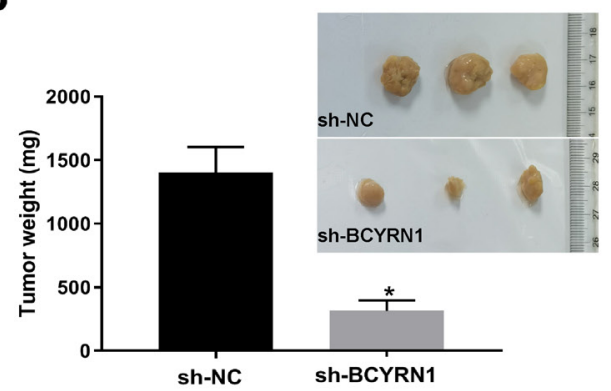

C

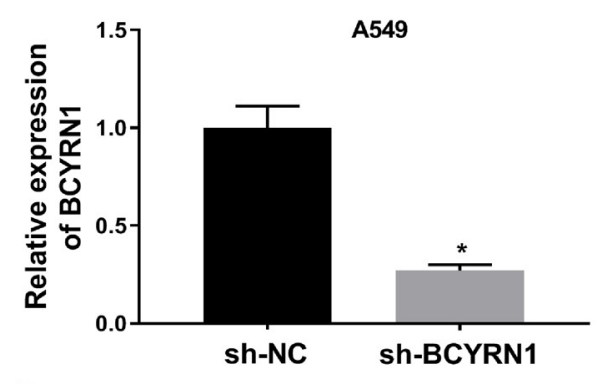

D

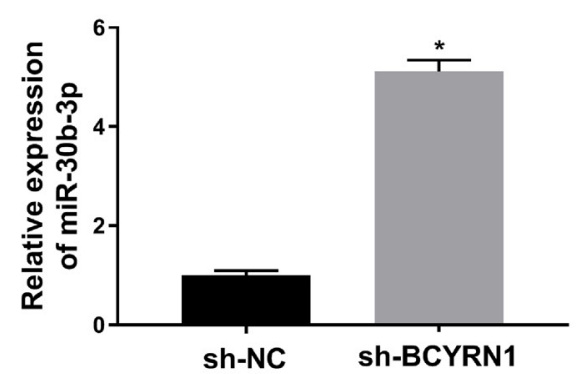

E
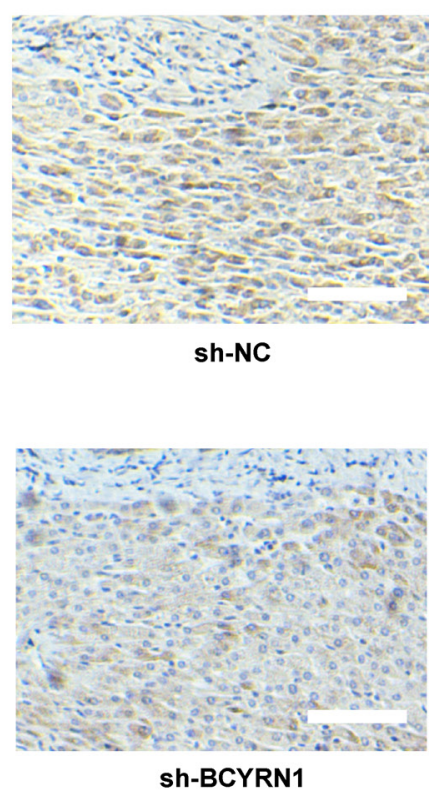

Figure 8. Knockdown of BCYRN1 inhibits tumor growth in nude mice in vivo. A) Tumor volumes were measured at the indicated time points (days 7, 14, 21, and 28). B) 28 days after the transfected cells were injected subcutaneously, the tumor was excised and weighed immediately. C, D) $q R T-P C R$ was used to detect the expression of BCYRN1 and miR-30b-3p in nude mouse xenograft tumors. (E) Immunohistochemical staining was used to identify the expression of ROCK1 in transplanted tumor tissues. The experiment was repeated three times. ${ }^{\star} \mathrm{p}<0.05,{ }^{\star *} \mathrm{p}<0.01,{ }^{\star * *} \mathrm{p}<0.001$. Scale bar $50 \mu \mathrm{m}$. 
between BCYRN1 and miR-30b-3p. Gu et al. [31] recently reported that DICER1 antisense RNA 1 is involved in the proliferation, invasion, and autophagy of osteosarcoma via the miR-30b/ATG5 axis. Furthermore, miR-30b overexpression suppresses cell proliferation and metastasis, via regulating the EMT process in gallbladder carcinoma [32]. In NSCLC, miR-30b is downregulated in tumors and associated with TNM stage, differentiation, and lymph node metastases. Besides, miR-30b suppresses viability, metastasis, and enhances apoptosis of the NSCLC cells by targeting the epidermal growth factor receptor [33]. Therefore, we inferred that miR-30b-3p may participate in the BCYRN1-mediated function in NSCLC. Subsequently, our data further demonstrated that ROCK1 can be directly targeted by miR-30b-3p. Accumulating studies supported that ROCK1 is overexpressed in human malignant tumors $[34,35]$. A high level of ROCK1 could enhance papillary thyroid carcinoma invasiveness [34]. Wang et al. [35] also unveiled that ROCK1 is notably elevated in osteosarcoma tumors and associated with tumor size, clinical stage, and distant metastasis. Meanwhile, ROCK1 could be directly targeted by miR-335 and participate in the osteosarcoma progression. And the involvement of ROCK1 in NSCLC also has been explored. Cui et al. [36] demonstrated that the miR-186/ROCK1 axis regulated NSCLC cell proliferation, migration, and invasion. Researchers also disclosed that miR-148b overexpression can repress cell proliferation and EMT via downregulating ROCK1 expression in NSCLC [37]. Similarly, we also found increased ROCK1 expression and decreased miR-30b-3p expression in NSCLC tissues and cells. The rescue-of-function assay further provided evidence that miR-30b-3p knockdown can abate the role of BCYRN1 in regulating the ROCK1 expression. In addition, si-BCYRN1-mediated metastasis repression in NSCLC cells was also undermined by the loss of miR-30b-3p. Collectively, our data indicated that BCYRN1 may be correlated with cell metastasis via the miR-30b-3p/ROCK1 axis.

We have also made some extensions to the present study. The previous studies have found that F-actin was involved in cell proliferation and motility, and its homeostasis could be regulated by ROCK1 (ROCK1 depolymerized F-actin) [38]. In the present study, ROCK1 expression was found to hamper F-actin expression, and miR-30b-3p was an upstream effector of ROCK1/F-actin. Increasing miR-30b-3p blocked the ROCK1/F-actin pathway-mediated lung cancer cells invasion, migration, as well as promoting cancer cells apoptosis. ROCK1/F-actin signal can trigger lung cancer cell mitochondrial apoptosis and delayed proliferation, whether miR-30b-3p is implicated in ROCK1-induced mitochondrial damage needs further research.

There are also some limitations to our study. For instance, we missed to detect the additional role of miR-30b-3p or ROCK1, which should be investigated in the future. Besides, the detailed function of the BCYRN1/miR-30b-3p/ROCK1 axis in NSCLC should be also addressed in vivo.
Taken together, our study first demonstrated that the BCYRN1/miR-30b-3p/ROCK1 axis was required for the metastasis of NSCLC, indicating that lncRNA BCYRN1 could be regarded as a potential therapeutic target for the treatment of NSCLC.

Acknowledgments: This work was supported by grants from Hospital fund of Shanghai Public Health Clinical Center (grant no. KY-GW-2020-13, KY-GW-2021-16); Projects of Medical Science and Technology Development Plan of Yancheng City (grant no. YK2018003); Shanghai Municipal Health Commission (grant no. 202040332).

\section{References}

[1] LOBB RJ, VAN AMERONGEN R, WIEGMANS A, HAM S, LARSEN JE et al. Exosomes derived from mesenchymal nonsmall cell lung cancer cells promote chemoresistance. Int J Cancer 2017; 141: 614-620. https://doi.org/10.1002/ijc.30752

[2] STEENDAM CM, DAMMEIJER F, AERTS J, CORNELISSEN R. Immunotherapeutic strategies in non-small-cell lung cancer: the present and the future. Immunotherapy 2017; 9: 507-520. https://doi.org/10.2217/imt-2016-0151

[3] HUARTE $M$. The emerging role of lncRNAs in cancer. Nat Med 2015; 21: 1253-1261. https://doi.org/10.1038/nm.3981

[4] PENG L, YUAN X, JIANG B, TANG Z, LI GC. LncRNAs: key players and novel insights into cervical cancer. Tumour Biol 2016; 37: 2779-2788. https://doi.org/10.1007/s13277015-4663-9

[5] BOLHA L, RAVNIK-GLAVAC M, GLAVAC D. Long Noncoding RNAs as Biomarkers in Cancer. Dis markers 2017; 2017: 1-14. https://doi.org/10.1155/2017/7243968

[6] RENGANATHAN A, FELLEY-BOSCO E. Long Noncoding RNAs in Cancer and Therapeutic Potential. Exp Biol Med 2017; 1008: 199-222. https://doi.org/10.1007/978-981-105203-3 7

[7] LI DY, BUSCH A, JIN H, EKATERINA C, JAROSLAV P et al. H19 induces abdominal aortic aneurysm development and progression. Circulation 2018; 138: 1551-1568. https:// doi.org/10.1161/CIRCULATIONAHA.117.032184

[8] HAN M, WANG S, FRITAH S, WANG X, ZHOU W et al. Interfering with long non-coding RNA MIR22HG processing inhibits glioblastoma progression through suppression of Wnt/ß-catenin signalling. Brain 2020; 143: 512-530. https:// doi.org/10.1093/brain/awz406

[9] WANG M, MAO C, OUYANG L, LIU Y, LAI W et al. Long noncoding RNA LINC00336 inhibits ferroptosis in lung cancer by functioning as a competing endogenous RNA. Cell Death Differ 2019; 26: 2329-2343. https://doi.org/10.1038/ s41418-019-0304-y

[10] CHEN J, WANG R, ZHANG K, CHEN LB. Long non-coding RNAs in non-small cell lung cancer as biomarkers and therapeutic targets. J Cell Mol Med 2014; 18: 2425-2436. https:// doi.org/10.1111/jcmm.12431

[11] WEI MM, ZHOU GB. Long Non-coding RNAs and Their Roles in Non-small-cell Lung Cancer. Genom Proteom Bioinf 2016; 14: 280-288. https://doi.org/10.1016/j.gpb.2016.03.007 
[12] YANG R, LIU N, CHEN L, JIANG Y, SHI Y et al. GIAT4RA functions as a tumor suppressor in non-small cell lung cancer by counteracting Uchl3-mediated deubiquitination of LSH. Oncogene 2019; 38: 7133-7145. https://doi.org/10.1038/ s41388-019-0909-0

[13] ZHU Q, LV T, WU Y, SHI X, LIU H et al. Long non-coding RNA 00312 regulated by HOXA5 inhibits tumour proliferation and promotes apoptosis in Non-small cell lung cancer. J Cell Mol Med 2017; 21: 2184-2198. https://doi.org/10.1111/ jcmm.13142

[14] HE R, ZHANG FH, SHEN N. LncRNA FEZF1-AS1 enhances epithelial-mesenchymal transition (EMT) through suppressing E-cadherin and regulating WNT pathway in non-small cell lung cancer (NSCLC). Biomed Pharmacother 2017; 95: 331-338. https://doi.org/10.1016/j.biopha.2017.08.057

[15] ZHANG Y, LI Y, WANG Q, ZHANG X, WANG D et al. Identification of an lncRNAmiRNAmRNA interaction mechanism in breast cancer based on bioinformatic analysis. Mol Med Report 2017; 16: 5113-5120. https://doi.org/10.3892/ mmr.2017.7304

[16] LI S, MEI Z, HU HB, ZHANG X. The lncRNA MALAT1 contributes to non-small cell lung cancer development via modulating miR-124/STAT3 axis. J Cell Physiol 2018; 233: 6679-6688. https://doi.org/10.1002/jcp.26325

[17] HU B, ZHANG H, WANG Z, ZHANG F, WEI H et al. LncRNA CCAT1/miR-130a-3p axis increases cisplatin resistance in non-small-cell lung cancer cell line by targeting SOX4. Cancer Biol Ther 2017; 18: 974-983. https://doi.org/ https://doi.org/.1080/15384047.2017.1385679

[18] WANG Y, BAI W, WANG M, YU T, ZHANG W. Long NonCoding RNA Brain Cytoplasmic RNA 1 Acts as an Oncogene and Regulates Cell Proliferation and Metastasis in NonSmall Cell Lung Cancer. J Nanosci Nanotechnol 2019; 19: 1978-1985. https://doi.org/10.1166/jnn.2019.16402

[19] ZHONG K, CHEN K, HAN L, LI B. MicroRNA-30b/c inhibits non-small cell lung cancer cell proliferation by targeting Rab18. BMC Cancer 2014; 14: 703. https://doi. org/10.1186/1471-2407-14-703

[20] LI J, SONG Y, WANG Y, LUO J, YU W. MicroRNA-148a suppresses epithelial-to-mesenchymal transition by targeting ROCK1 in non-small cell lung cancer cells. Mol Cell Biochem 2013; 380: 277-282. https://doi.org/10.1007/s11010013-1682-y

[21] MONTES M, LUBAS M, ARENDRUP FS, MENTZ B, ROHATGI $\mathrm{N}$ et al. The long non-coding RNA MIR31HG regulates the senescence associated secretory phenotype. Nat Commun 2021; 12: 1-17. https://doi.org/10.1038/s41467021-22746-4

[22] MAO C, WANG X, LIU Y, WANG M, YAN B et al. A G3BP1interacting lncRNA promotes ferroptosis and apoptosis in cancer via nuclear sequestration of p53. Cancer Res 2018; 78: 3484-3496. https://doi.org/10.1158/0008-5472.CAN-173454

[23] ELGUINDY MM, MENDELL JT. NORAD-induced Pumilio phase separation is required for genome stability. Nature 2021; 595: 303-308. https://doi.org/10.1038/s41586-02103633-w
[24] ZHANG CG, YIN DD, SUN SY, HAN L. The use of lncRNA analysis for stratification management of prognostic risk in patients with NSCLC. Eur Rev Med Pharmacol Sci 2017; 21: 115-119.

[25] TIAN FM, MENG FQ, WANG XB. Overexpression of longnoncoding RNA ZFAS1 decreases survival in human NSCLC patients. Eur Rev Med Pharmacol Sci 2016; 20: 5126-5131.

[26] WU Y, HUANG C, MENG X, LI J. Long Noncoding RNA MALAT1: Insights into its Biogenesis and Implications in Human Disease. Curr Pharm Des 2015; 21: 5017-5028. https://doi.org/10.2174/1381612821666150724115625

[27] GU L, LU L, ZHOU D, LIU Z. Long Noncoding RNA BCYRN1 Promotes the Proliferation of Colorectal Cancer Cells via Up-Regulating NPR3 Expression. Cell Physiol Biochem 2018; 48: 2337-2349. https://doi.org/10.1159/000492649

[28] PENG J, HOU F, FENG J, XU SX, MENG XY. Long noncoding RNA BCYRN1 promotes the proliferation and metastasis of cervical cancer via targeting microRNA-138 in vitro and in vivo. Oncol Lett 2018; 15: 5809-5818. https:// doi.org/10.3892/ol.2018.8015

[29] REN H, YANG X, YANG Y, ZHANG X, ZHAO R et al. Upregulation of LncRNA BCYRN1 promotes tumor progression and enhances EpCAM expression in gastric carcinoma. Oncotarget 2018; 9: 4851-4861. https://doi.org/10.18632/ oncotarget. 23585

[30] HU T, LU YR. BCYRN1, a c-MYC-activated long non-coding RNA, regulates cell metastasis of non-small-cell lung cancer. Cancer Cell Int 2015; 15: 36. https://doi.org/10.1186/ s12935-015-0183-3

[31] GU Z, HOU Z, ZHENG L, WANG X, WU L et al. LncRNA DICER1-AS1 promotes the proliferation, invasion and autophagy of osteosarcoma cells via miR-30b/ATG5. Biomed Pharmacother 2018; 104: 110-118. https://doi.org/10.1016/j. biopha.2018.04.193

[32] WANG N, XIANG X, CHEN K, LIU P, ZHU A. Targeting of NT5E by miR-30b and miR-340 attenuates proliferation, invasion and migration of gallbladder carcinoma. Biochimie 2018; 146: 56-67. https://doi.org/10.1016/j.biochi.2017.10.027

[33] QI Z, ZHANG B, ZHANG J, HU Q, ZHU C. MicroRNA-30b inhibits non-small cell lung cancer cell growth by targeting the epidermal growth factor receptor. Neoplasma 2018; 65: 192-200. https://doi.org/10.4149/neo_2018_170217N118

[34] LUO D, CHEN H, LI X, LU P, LONG M et al. Activation of the ROCK1/MMP-9 pathway is associated with the invasion and poor prognosis in papillary thyroid carcinoma. Int J Oncol 2017; 51: 1209-1218. https://doi.org/10.3892/ ijo. 2017.4100

[35] WANG Y, WANG N, ZENG X, SUN J, WANG G et al. MicroRNA-335 and its target Rock1 synergistically influence tumor progression and prognosis in osteosarcoma. Oncol Lett 2017; 13: 3057-3065. https://doi.org/10.3892/ol.2017.5818

[36] CUI G, CUI M, LI Y, LIANG Y, LI W et al. MiR-186 targets ROCK1 to suppress the growth and metastasis of NSCLC cells. Tumour Biol 2014; 35: 8933-8937. https://doi. org/10.1007/s13277-014-2168-6 
[37] LUO H, LIANG C. MicroRNA-148b inhibits proliferation and the epithelial-mesenchymal transition and increases radiosensitivity in non-small cell lung carcinomas by regulating ROCK1. Exp Ther Med 2018; 15: 3609-3616. https://doi. org/10.3892/etm.2018.5845
[38] ZHANG X, XU L, YANG T. miR-31 modulates liver cancer HepG2 cell apoptosis and invasion via ROCK1/F-Actin Pathways. Oncotargets Ther 2020; 13: 877. https://doi. org/10.2147/OTT.S227467 